\title{
T h h ema \\ Des pratiques épistémiques d'ingénierie sur le processus de conception technique pour instituer une communauté discursive interdisciplinaire scolaire à l'école obligatoire
}

\author{
Patrick Roy, Haute école pédagogique Fribourg
}

Cet article consiste en une réflexion conceptuelle d'ordre épistémologique et didactique sur un enseignement de la technologie centré sur le processus de conception technique. En vue de construire une communauté discursive interdisciplinaire scolaire dans ce domaine, nous convions les enseignant.e.s à faire acquérir aux élèves six pratiques épistémiques d'ingénierie : définir un problème technique, générer et sélectionner des solutions en recourant à une approche systématique et itérative, analyser et modéliser, expérimenter et évaluer, communiquer et prendre des décisions. Ces pratiques discursives interdisciplinaires caractérisant la pensée design des élèves sont cohérentes avec les pratiques sociotechniques de référence et jouent un rôle central dans le développement d'une culture technique authentique.

\section{Introduction}

Notre environnement quotidien comporte une multitude d'objets qui ont été préalablement conçus au moyen de processus de conception issus de domaines professionnels extrêmement variés, allant de secteurs techniques pointus à des secteurs artistiques (Bonnardel, 2009). Dans le monde de l'ingénierie, la conception est de loin l'activité centrale des ingénieur.e.s (Simon, 1995). C'est pourquoi plusieurs systèmes éducatifs à travers le monde promeuvent dans leur curriculum un enseignement de la technologie centré sur le Processus de Conception Technique $(\mathrm{PCT})^{1}$; ce processus étant désigné dans le monde anglo-saxon sous le vocable d'" Engineering Design Process » (Berland et al., 2014; Daugherty, 2012 ; Dearing et Daugherty, 2004 ; Pearson et Young, 2002 ; Wicklein, 2006). Les avantages d'opter pour un enseignement de la technologie centré sur le PCT à l'école obligatoire sont nombreux. Parmi ceux-ci, on peut mentionner le développement de la pensée design ("design thinking ") (Bonnardel, 2006, 2009 ; Didier, 2017) qui est au cœur des PCT (Sheppard et al., 2009, p. 100) :

Engineering design involves a way of thinking that is increasingly referred to as design thinking : a high level

of creativity and mental discipline as the engineer tries to discover the heart of the problem and explore

beyond the solutions at easy reach.

Il importe de souligner que le concept de pensée design recouvre une pluralité de significations dans la documentation scientifique et que les cadres théoriques pour la caractériser sont très nombreux. Johansson-Sköldberg, et al. (2013) relèvent que cette pensée peut être appréhendée sous l'angle de la conception d'artéfacts, d'une activité de résolution de problèmes ou d'une pratique réflexive. Comme le montre la définition de Sheppard, et al. (2009) plus haut, la pensée design est une pensée qui revêt une dimension créative. En tant que concepteurs ou conceptrices, les ingénieur.e.s sont appelé.e.s à développer des artéfacts à la fois novateurs et adaptés aux besoins des utilisateurs et utilisatrices, et à faire preuve de créativité (Bonnardel, 2006). Ainsi, pour s’engager dans un PCT, ils ou elles doivent disposer non seulement de connaissances techniques et d'une expertise dans leur domaine, mais aussi d'un potentiel de créativité (Borillo et Goulette, 2002)2. Rauth, et al. (2010, p. 1) reprennent la définition de Woodman (1993) pour dire que la créativité en matière de conception conduit à

1 Pearson et Young (2002, p. 58) affirment : « Design is the central component of the practice of engineering and a key element in technology education ".

2 Dans les définitions retenues par les auteurs ou autrices pour caractériser ce qu'est un PCT, la composante créative du PCT n'est toutefois pas toujours mise en exergue (Bonnardel, 2006). Par exemple, pour Demailly et Lemoigne (1986, p. 435-436) : "Concevoir (to design), c'est dessiner, exprimer un dessein par un dessin ou par une forme ou par un système de symboles [...] c'est créer, ou construire, quelque modèle symbolique à l'aide duquel on inferrera ensuite le réel ». De même pour Simon (1995, p. 246), il s'agit de " concevoir des objets, des processus, des idées pour accomplir des buts, et montrer comment ces objets, processus ou idées peuvent être réalisés ». 
une meilleure compréhension de la capacité humaine à résoudre des problèmes complexes et à créer «a valuable, useful new product, service, idea, procedure, or process by individuals working together in a complex social system ". La majorité des auteurs et autrices qui s'intéresse au PCT s'accorde à dire que la créativité consiste en la capacité à avoir une idée ou à réaliser une production à la fois nouvelle et adaptée au contexte dans lequel elle se manifeste (Bonnardel, 2009). Pour Bonnardel (2006), la créativité d'un concepteur ou d'une conceptrice est particulièrement interpelée dans des activités de conception non routinières qu'elle qualifie également par l'expression de " creative design ». À la suite de Bonnardel (2009, p. 8), nous concevons la créativité au sein d'un processus de conception comme étant :

La capacité à générer une idée, une solution ou une production qui soit à la fois nouvelle et adaptée à la situation et, dans certains cas, considérée comme ayant une certaine utilité ou valeur. Les productions en question peuvent être de nature variée. Dans le cas des activités de conception, il s'agit principalement d'objets de la vie quotidienne et d'objets techniques.

La pensée design fait partie des modes de pensée nécessaires pour évoluer dans un monde hautement technologique (Bonnardel, 2009). Par ailleurs, plusieurs chercheur.e.s soulignent que l'enseignement de la technologie centré sur le PCT favorise l'interdisciplinarité scolaire dans le domaine des MINT33 (Daugherty, 2012 ; Householder et Hailey, 2012 ; Roth, 2001), le développement de modes de pensée génériques (comme la pensée créative qui est intimement liée à la pensée design, ou d'autres modes de pensée comme la pensée critique ou conceptuelle) (Doppelt et al., 2008 ; Hmelo et al., 2000 ; Moore et al., 2014), le développement de compétences en résolution de problèmes (Eide et al., 2002 ; Fortus et al., 2005 ; Kolodner et al., 2003 ; Moore et al., 2014), l'engagement des élèves dans les apprentissages disciplinaires (Fortus et al., 2004 ; Roth, 2001), l'acquisition d'un vocabulaire spécifique au domaine de l'ingénierie (Aguirre-Muñoz et Pantoya, 2016 ; Kelly et al., 2017), l'appropriation de connaissances-métier de l'ingénieur·e (Capobianco et al., 2015 ; Rynearson, 2016) ou encore l'intérêt pour les métiers et professions du monde technique (Cunningham et Hester, 2007 ; Mehalik et al., 2008).

Pour développer une culture technique qui se veut la plus authentique possible à l'école obligatoire, Lebeaume (2001) invoque la problématique de la référence curriculaire et reprend le concept de pratiques sociales de référence proposé antérieurement par Martinand (1982) afin de questionner le sens et les finalités des activités technologiques scolaires par rapport aux pratiques de la technologie s'exerçant dans la société, en particulier celles des ingénieur·e.s. À ce sujet, Lebeaume (2001, p. 129) précise que l'enjeu de l' "authenticité, qui s’oppose à fausseté, est alors conceptualisée en termes de référence et de pratiques sociales de référence par Martinand (1982) ». Depuis son élaboration, le concept de pratiques sociales de référence s'est progressivement transformé en celui de pratiques sociotechniques de référence renforçant ainsi le caractère technique des pratiques de référence pour l'enseignement de la technologie et la dimension sociale des actions techniques (Lebeaume, 2001). Si les pratiques sociotechniques de référence permettent de garantir une certaine authenticité des activités scolaires par rapport aux activités industrielles (Martinand, 2013), il ne s'agit pas pour autant de reproduire ces pratiques intégralement au niveau de la classe ; l'école poursuit d'autres finalités que celles du monde technique. Ainsi, dans une perspective de transposition didactique et de référenciation à des pratiques sociales, le qualificatif d'authenticité, lorsqu'il est associé à un objet scolaire, en particulier à une pratique scolaire de la technologie, véhicule l'idée que cet objet est bel et bien " référé " à une pratique sociotechnique de référence. Cela signifie qu'une pratique scolaire de la technologie n'est pas et ne se doit pas d'être une pratique identique à celle pouvant être exercée par un concepteur ou une conceptrice dans sa pratique professionnelle. Le caractère authentique d'une pratique scolaire de la technologie signifie par ailleurs qu'il importe que cette pratique ne soit pas issue d'une culture scolaire distante, voire épistémologiquement incohérente avec une culture technique définie par des pratiques sociotechniques de référence.

Dès lors, se posent certaines questions fondamentales concernant la transposition didactique des pratiques sociotechniques de référence exercées par des ingénieur.e.s en des pratiques scolaires d'ingénierie exercées par des élèves : quelles sont les principales pratiques sociotechniques de référence en lien avec les PCT qui méritent d'être considérées à l'école obligatoire (enseignement primaire et secondaire) ? Comment ces pratiques se transposent-elles en classe ? En d'autres mots, quelles sont les pratiques d'ingénierie authentiques qu'il faut développer chez les élèves? C'est à ces questions que nous nous attardons dans cet article. Il consiste en une

3 L'acronyme MINT désigne l'ensemble des disciplines Mathématiques, Informatique, sciences Naturelles et Technique et se réfere au STEM dans le monde anglo-saxon. 
réflexion conceptuelle ${ }^{4}$ d'ordre épistémologique et didactique sur l'enseignement de la technologie centré sur le PCT. L'objectif est de développer ce que Cunningham et Kelly (2017a, 2017b, 2019) appellent les Pratiques Epistémiques d'Ingénierie (PEI) ("Epistemic Practices of Engineering») afin d'éclairer l'élaboration des curricula, la formation initiale et continue des enseignant.e.s de l'école obligatoire et la recherche dans le domaine de l'éducation technique.

Cet article est divisé en quatre parties. Dans la première partie, nous définissons le concept de PEI en nous référant aux concepts de pratiques épistémiques et de pratiques sociotechniques de référence, et nous montrons comment les PEI se rattachent à l'idée de construire une communauté discursive interdisciplinaire scolaire en technologie. Dans la seconde partie, nous dégageons six processus cognitifs fondamentaux (que nous désignons par les PEI) pour caractériser la pensée design. Ces processus sont récurrents dans la documentation scientifique portant sur le PCT. Dans la troisième partie, nous développons ces pratiques sur les plans épistémologique et didactique. La quatrième partie est dédiée à la conclusion.

\section{Les pratiques épistémiques d'ingénierie: des pratiques discursives interdisciplinaires pour caractériser les PCT chez les élèves}

L'ingénierie consiste en « a fundamental human process that has been practiced from the earliest days of civilization " (Petroski, 1996, p. 2). Au fil du temps, les méthodes utilisées par les humains pour résoudre des problèmes et fabriquer des artéfacts en vue de répondre à divers besoins ont été formalisées dans le monde des ingénieur.e.s en ce que l'on appelle aujourd'hui le PCT (Smith, 2006). La documentation scientifique fait état d'une panoplie de définitions qui rendent compte de ce processus. Celles-ci different selon les disciplines et les projets sur lesquels les concepteurs et les conceptrices travaillent (Berland et al., 2014). Considérons la définition assez complète du Conseil d'accréditation américain pour l'ingénierie et la technologie (The accreditation board for Engineering and Technology) pour définir ce processus :

Engineering design is the process of devising a system, component, or process to meet desired needs. It is a decision-making process in which the basic sciences and mathematics and engineering sciences are applied to convert resources optimally to meet a stated objective. Among the fundamental elements of the design process are the establishment of objectives and criteria, synthesis, analysis, construction, testing, and evaluation. It is essential to include a variety of realistic constraints, such as economic factors, safety, reliability, aesthetics, ethics, and social impact. (Oakes et al., 2002, p. 339)

Nous dégageons de cette définition deux considérations importantes. La première est que le PCT est un processus interdisciplinaire. Il implique au moins la contribution des sciences de l'ingénierie, des sciences de la nature et des mathématiques. ${ }^{5}$ C'est pourquoi ce processus, lorsqu'il est transposé à la classe, constitue pour plusieurs chercheur.e.s une approche interdisciplinaire de prédilection (Daugherty, 2012 ; Hacioğlu et al., 2017 ; Householder et Hailey, 2012 ; Roth, 2001) ${ }^{6}$. La seconde considération est que le PCT sollicite les ingénieur·e.s à mobiliser des processus cognitifs spécifiques : établir des objectifs et des critères, synthétiser, analyser, construire, tester, évaluer et prendre des décisions. Ces processus cognitifs caractérisent en quelque sorte la pensée design des ingénieur.e.s.

4 La plupart des travaux cités dans cette réflexion conceptuelle sont en grande partie des travaux anglo-saxons. Ils découlent d'une recension des écrits scientifiques dans la banque de données ERIC pour la période 2000-2020 où l'expression Engineering Design Process figurait dans le titre ou le résumé de l'article. En conséquence, nous exprimons ici une vision anglo-saxonne de l'éducation technologique à laquelle nous adhérons.

5 En suivant le Conseil d'accréditation américain pour l'ingénierie et la technologie, s'ajoutent également de potentielles contributions de sciences sociales : esthétique, éthique, et toutes celles qui permettent de prendre en compte un "impact social ".

6 À titre d'exemple, Hacioğlu et al. (2017) proposent d'engager les élèves dans l'approche pédagogique Engineering Design Based Science Education (EDBSE). L'EDBSE « is an educational approach that involves the integration of STEM disciplines, which handle the inquiry and engineering design together in order to enable students to acquire the targeted behaviors and aim to generate solutions for engineering design problems that form the real-life context within the scope of the process of engineering design » (Hacioğlu et al., 2017, p. 653). 
Dans une perspective de transposition didactique, des pratiques des ingénieur.e.s aux pratiques des élèves en se centrant sur les processus cognitifs en jeu dans les PCT, Cunningham et Kelly (2017a, 2017b, 2019) ont développé, sur la base d'études empiriques en ingénierie dans les milieux professionnels et scolaires, le concept de Pratiques Épistémiques d'Ingénierie (PEI) ("Epistemic Practices of Engineering »). Les pratiques épistémiques sont des manières d'agir socialement organisées au sein d'un groupe afin de proposer, communiquer, évaluer et légitimer la construction de nouvelles connaissances (Kelly et Licona, 2018). La définition du concept de pratiques épistémiques proposée par Cunningham et Kelly (2017a) dans le tableau 1 (en annexe) met en exergue deux aspects très importants de ces pratiques. Le premier est que ces pratiques sont par essence des pratiques discursives (langagières) en raison de leur caractère interactionnel, contextuel et intertextuel (caractéristiques 1 à 3). Le second aspect est que ces pratiques ne sont pas des pratiques discursives génériques détachées des savoirs et valables pour tous les domaines de connaissance. Elles constituent des pratiques fondées sur des savoirs formalisés comme le mettent en évidence les caractéristiques 1, 2 et 4 de cette définition. Comme le rappelle Combarnous (1984, p. 29), la technicité est « indissociable de la capacité de constituer des associations de connaissances raisonnées et empiriques, toutes éprouvées par la pratique, qui assurent l'efficacité des actions ". C'est pourquoi on dit que ce sont des pratiques épistémiques. Les PEI sont donc des pratiques discursives spécifiques de l'activité d'ingénierie qui se situent au niveau de la classe et qui sont épistémologiquement cohérentes avec les pratiques sociotechniques de référence des ingénieur.e.s (Lebeaume, 2001). Ces dernières sont bel et bien des pratiques discursives fondées sur des savoirs formalisés. En effet, pour être reconnu.e.s comme membres d'une communauté d'ingénieur.e.s, ces derniers et ces dernières doivent adhérer à un ensemble de pratiques, de valeurs, de croyances, d'attitudes et de manières d'être de ce monde façonné par un discours spécialisé de cette communauté épistémique (Kelly, 2016). Ainsi, les ingénieur·e·s, du moins à l'intérieur d'une même spécialité, font partie d'une même communauté de pratique (Wenger, 1998) et partagent, entre autres, des pratiques discursives communes (Kelly et Cunningham, 2019). Dans la mise en ouvre d'un PCT, ils ou elles sont appelé.e.s à mobiliser des savoirs formalisés. À ce sujet, Dym et al. (2005, p. 108) relèvent que le PCT implique la mobilisation de concepts spécifiques de la conception :

Designers think about design processes when they begin to sketch and draw the objects they are designing. A complete representation of designed objects and their attributes requires a complete representation of design concepts - e.g., design intentions, plans, behavior, and so on - that are harder to describe or represent than are physical objects.

Dans la communauté scientifique, les pratiques discursives participent à la construction incessante des savoirs des sciences de l'ingénierie, car elles soutiennent la production, l'explicitation et l'évaluation des connaissances, et leur mise en texte dans les publications scientifiques (Kelly et Cunningham, 2019). Comme le PCT implique soit la mobilisation de savoirs de plusieurs disciplines ou la mise en perspectives des regards d'une équipe de collaborateurs ou de collaboratrices de plusieurs domaines professionnels, Lindberg, et al. (2009) considèrent ce processus comme une méthodologie métadisciplinaire, c'est-à-dire " a precious methodology for interdisciplinary creative work as it specifically complements mono-disciplinary thinking ». Ainsi, on peut dire que les PEI sont des pratiques interdisciplinaires (Kelly et Licona, 2018) au sens de Fourez et al. (2002, p. 62) et qu'elles conduisent à "l'utilisation, l'association et la coordination de disciplines appropriées, dans une approche intégrée des problèmes ». Ces pratiques exigent des élèves de mobiliser, de manière intégrée, des concepts et processus des sciences de l'ingénierie et d'autres disciplines comme les sciences et les mathématiques.

Ces constats ont une conséquence importante pour la classe, en particulier pour le développement d'une culture technique pour tous et pour toutes. À l'école, l'apprentissage des prémisses d'une activité d'ingénierie implique de développer chez les élèves un répertoire de pratiques discursives spécifiques de cette communauté (Kelly, 2016 ; Kelly et Green, 1998 ; Wickman, 2004). Dans les termes de Bernié et ses collègues (2002 ; Jaubert et al., 2004), il s'agit pour l'enseignant.e de construire une Communauté Discursive Disciplinaire Scolaire (CDDS). L'idée centrale de ce concept est que le développement d'une culture disciplinaire repose sur une acculturation des élèves à des manières de penser, de parler et d'agir spécifiques de cette discipline, en l'occurrence ici la technologie. Jaubert, et al. (2004, p. 85) soulèvent par ailleurs la nécessité de distinguer " communauté de pratique » et " communauté de discours (discursive) », cette dernière met l'accent sur l'importance des significations partagées :

Une communauté de discours n'est pas l'équivalent d'une communauté de pratiques, et, si l'on peut considérer les deux expressions comme aussi insuffisantes l'une que l'autre pour enfermer ce qu'y mettent les chercheurs qui s'y intéressent, le choix du terme "discursif" indique suffisamment l'option de base : apprendre est irréductible au partage de pratiques, et la relation apprentissage / développement suppose la prise en compte de la genèse des significations, qui n'est pas de l'ordre de l'immédiateté. 
La composante discursive d'une CDDS comporte deux dimensions : une dimension culturelle véhiculée par le contenu des discours, conjuguée à une dimension sociale (de communication) véhiculée par les modalités de transmission des discours avec des systèmes de signes spécifiques. C'est pourquoi les composantes épistémique et sociale des PEI vont de pair. Si nous admettons que le PCT est un processus interdisciplinaire qui mobilise de manière intégrée les "paradigmes disciplinaires " (Astolfi, 2008) 7 ou les " raisonnements extraordinaires 8 (Orange, 2018) de plusieurs disciplines (les sciences de l'ingéniénierie, les mathématiques, les sciences de la nature, les sciences humaines et sociales, etc.) en établissant des liens de complémentarité, de coopération, d'interpénétrations ou d'actions réciproques, entre les composantes de ces disciplines (Fourez, 1997), nous pouvons dire que les PEI participent à la construction d'une Communauté Discursive Interdisciplinaire Scolaire (CDIS).

\section{Les pratiques épistémiques d'ingénierie : à la recherche des processus cognitifs fondamentaux en jeu dans les PCT}

Les processus cognitifs utilisés par les chercheur-e.s pour caractériser les PCT qui s'opèrent dans les pratiques professionnelles des ingénieur·e.s ou celles des élèves à l'école obligatoire sont très diversifiés et ne font pas l'objet d'un consensus. Néanmoins, certains processus apparaissent de manière récurrente dans la littérature. Sans vouloir rendre compte de manière exhaustive des travaux ${ }^{9}$ qui se sont intéressés aux processus cognitifs caractérisant la pensée design des ingénieur.e.s et des élèves, nous en rapportons ici quelques-uns dont plusieurs ont été recensés dans le cadre d'une revue de littérature réalisée par Grubbs et al. (2018). Welch et Lim (2000) ont caractérisé la pensée stratégique de concepteurs et conceptrices novices (élèves de la 7e année) dans des PCT non encadrés sur la base de cinq processus cognitifs émergents : understanding the problem, generate possible solutions, modeling a possible solution, building a solution, evaluation. Afin de promouvoir les PCT dans les cours du domaine MINT des écoles secondaires américaines, Hynes et al. (2011) ont élaboré un modèle de PCT en neuf étapes (identify need or problem, research need or problem, develop possible solution(s), select best possible solution(s), construct a prototype, test and evaluate solution(s), communicate the solution(s), redesign, completion (leaves the cycle)) qui a été adopté et recommandé par le National Center for Engineering and Technology Education comme modèle emblématique pour opérationnaliser le PCT en classe P-12 (figure 1). En outre, ce modèle a été utilisé ultérieurement par Wilson et al. (2013) pour étudier l'activité cognitive de lycéens et lycéennes engagé.e.s dans la résolution de problèmes d'ingénierie authentiques.

\section{Figure 1}

Model of the Engineering Design Process (Hynes et al., 2011)

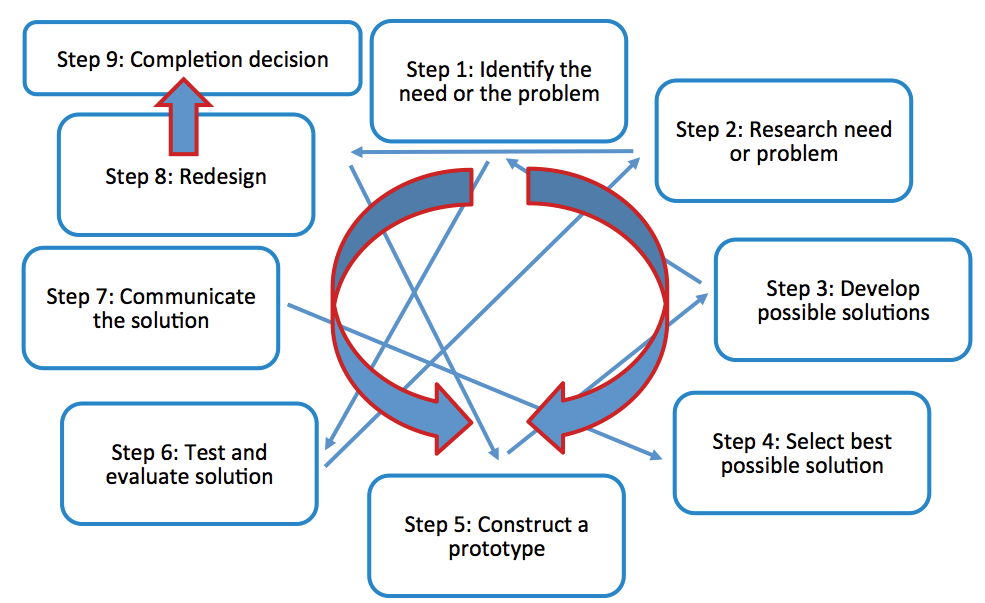

\footnotetext{
7 À ce sujet, Astolfi (2008, p. 32) affirme qu'à l'école «il n’y a pas de véritables savoirs sans accès aux paradigmes disciplinaires, chacun étant original par le fonctionnement du langage qui y prévaut et par les formes de raisonnement qui y sont valides ».

8 Il s'agit de manières extraordinaires de penser qui ne peuvent être produites et mobilisées dans les discours de sens commun.

9 Les travaux de Bonnardel et de Choulier dressent un état des lieux complet sur les processus cognitifs des élèves engagé.e.s dans un PCT.
} 
Moore et al. (1995) ont réalisé une analyse de contenu de sept manuels d'introduction à la conception technique couramment utilisés dans la formation des ingénieur.e.s aux États-Unis et ont identifié huit processus cognitifs communs pour décrire les PCT véhiculés dans ces manuels : problem definition, information gathering, generating ideas, modelling, feasibility analysis, evaluation, decision making, teamwork/communication. Ces processus cognitifs ont été utilisés ultérieurement dans les travaux de Mentzer (Mentzer, 2011b, 2014 ; Mentzer et al., 2015) pour caractériser la pensée design d'élèves du secondaire, et dans ceux d'Atman et al. (2007) pour caractériser cette même pensée, mais dans une perspective comparative, chez des élèves du secondaire, des étudiant.e.s en ingénierie de plusieurs degrés et des ingénieur.e.s expert.e.s. En comparant le modèle de PCT classique d'Eide et al. (2002) avec le PCT proposé dans le Standard 8 for tecnological literacy Design Process for grades 9-12, Hailey et al. (2005, p. 25) mettent en exergue certaines différences significatives entre les PCT pouvant être conduits par des ingénieur.e.s expert.e.s et des élèves du secondaire (tableau 2 en annexe). Si la définition du problème, la génération et la sélection de solutions, l'analyse et la modélisation, ainsi que la communication apparaissent comme étant des processus cognitifs communs aux deux communautés, les PCT conduits par les ingénieur.e.s expert·e.s impliquent néanmoins des tâches d'analyse et de modélisation plus poussées. Ces travaux nous conduisent à retenir six PEI pour la classe. Il est important de souligner que pratiques ne sont pas mutuellement exclusives et ne se déploient pas linéairement selon un ordre chronologique comme nous les développons dans la section suivante.

\section{Six pratiques épistémiques d'ingénierie}

\subsection{Définir un problème technique}

Burghardt (1999, p. 1) considère les ingénieur.e.s comme des « creative problem solvers, often imagining and designing new technologies as a means to solve problems ". La résolution de problèmes fait partie de ce que Dugger (1994, p. 7) appelle le noyau philosophique (" philosophical nucleus ») de la culture technique. Tout PCT s'amorce avec la définition d'un problème. Pour engager les élèves dans une véritable activité de résolution de problèmes, Lewis (2005) suggère d'intégrer les défis en matière de conception technique ("Engineering Design Challenges ») aux PCT. Les problèmes présentés aux élèves peuvent être de deux types. Il peut s'agir de problèmes bien définis ("Well-structured problems ") ou de problèmes " mal définis » ou " mal structurés» ("Ill-structured problems ") appelés aussi des problèmes ouverts ("Open-ended problems") (Jonassen, 1997). Les problèmes bien définis appellent élèves à mettre en application des contenus spécifiques dans le cadre d'une démarche prédéfinie. Ils concernent ce que Bonnardel (2009, p. 8) appelle les activités de conception routinières dans lesquelles « le produit à concevoir étant peu différent des produits développés antérieurement, le concepteur peut adapter un schéma prédéfini afin de traiter le problème considéré. ». Pour ce type de problème, l'enseignant.e peut par exemple fournir un cahier des charges qui présente une gamme de fabrication très détaillée qu'il s'agit de suivre à la lettre comme une recette de cuisine. Quant aux problèmes "mal définis" ou "mal structurés", ils contiennent des informations manquantes et appellent les élèves à identifier les objectifs et les stratégies pour les résoudre (Ertmer et al., 2009). Ils concernent ce que Bonnardel (2009, p. 8) appelle les activités de conception non routinières. Elle souligne que dans de telles activités :

Aucun schéma prédéfini ne peut être adapté au problème et le concepteur doit faire preuve d'une certaine créativité. Ce dernier type d'activités de conception aboutit à des objets nouveaux, notamment par une extension de produits préexistants ou par la combinaison de traits issus de différents produits existant de façon à en constituer un nouveau.

Les activités de conception non routinières engagent les élèves dans l'innovation plutôt que dans la rénovation si l'on reprend le propos d'Elmar Mock, l'un des deux inventuers de la montre Swatch (lauréat du prix Gaïa), qui explique bien l'importance de comprendre les enjeux et les différences entre ces deux concepts. Les PCT comportant des problèmes «mal définis » ou «malstructurés» sont de loin les plus appropriés (Lewis, 2005, p. 49) : "Design appropriate for technology education is characterized by open-ended problems where the designer bridges the gap between past experiences and the current problem to be solved; one method of achieving this transition is through engineering design challenges ». Ils remettent en question l'enseignement traditionnel basé sur la transmission des connaissances, présentent des défis stimulants pour les élèves et favorisent leur développement intellectuel (Prince et Felder, 2006). Dans de tels problèmes, le concepteur ou la conceptrice dispose au départ d'une représentation mentale incomplète et imprécise de l'artéfact à concevoir (Bonnardel, 2009). 
Pour les ingénieur.e.s, définir un problème technique, c'est d'abord et avant tout clarifier un besoin à satisfaire en termes de fonctions ${ }^{10}$ et de contraintes. ${ }^{11}$ Le choix définitif d'une solution technique est un compromis qui dépend de la valeur accordée à ces différentes contraintes. Définir un problème technique, c'est aussi spécifier un ou des objectif(s) de performance, questionner ${ }^{12}$ des aspects du problème qui ne sont pas clairs, distinguer entre des données pertinentes et non pertinentes, identifier des données manquantes et prévoir comment les repérer dans le cadre d'une recherche documentaire avant d'entamer l'élaboration d'une solution, planifier une démarche permettant de construire des solutions hypothétiques, ou encore identifier des connaissances et des savoirs à mobiliser. Contrairement aux étudiant.e.s en ingénierie et aux ingénieur.e.s novices, les ingénieur.e.s expérimenté.e.s consacrent davantage d'effort et de temps à la définition du problème avant de s'engager dans la recherche de solutions (Atman et al., 2007 ; Dorst, 2011 ; Mentzer et al., 2015). Les expert·e.s ont le souci de recueillir des informations leur permettant de se donner une compréhension approfondie et multifactorielle du problème (Bogusch et al., 2000). Concrètement, la définition d'un problème technique peut se faire au moyen d'un cahier des charges fonctionnel dont les caractéristiques sont exposées dans le tableau 3 (en annexe).

\subsection{Générer et sélectionner des solutions en recourant à une approche systématique et itérative}

Le PCT est essentiellement une affaire de production, d'évaluation et de réalisation d'idées (Sheppard et al., 2009). Il conduit généralement à des solutions multiples dont certaines sont sélectionnées, et par la suite, optimisées en fonction de variables, critères et contraintes spécifiques (Kelly et Cunningham, 2019). La génération et la sélection de solutions consistent en l'organisation d'un ensemble de conceptions partielles (de chacune des composantes d'un objet technique) en vue de répondre à un problème défini. C'est ce que Dym (1994, p. 28) appelle la tâche de synthèse :

Synthesis is the task of assembling a set of primitive design elements or partial designs into one or more configurations that clearly and obviously satisfy a few key objectives and constraints. Synthesis is often considered as the task most emblematic of the design process.

Concernant la génération des solutions créatives, elle implique des étapes spécifiques telles que l'« incubation " au cours de laquelle de nombreuses associations d'idées sont créées de façon inconsciente et l' « illumination " qui correspond à la prise de conscience soudaine d'une idée intéressante (Gelb, 1996). La génération des solutions créative se concrétise par la conception et la fabrication de prototypes et mobilise la composante pensée divergente de la pensée design pour produire de nombreuses idées créatives (Dym et al., 2005). Lors de la génération des solutions, les ingénieur.e.s expérimenté.e.s recourent à des stratégies de conception efficaces et systématiques. Contrairement aux novices qui se focalisent souvent sur une solution spécifique sans envisager d'autres alternatives (Mentzer et al., 2015), les ingénieur·e.s expérimenté.e-s sont capables de concevoir et de fabriquer des prototypes en gérant simultanément, dans une perspective systémique, un ensemble de variables, critères et contraintes spécifiques (Dorst, 2011). Une stratégie accompagne ce processus : le remue-méninges (individuel, mais le plus souvent collectif) par lequel les concepteurs ou les conceptrices sont appelée.es à formuler des idées dans un court laps de temps, et au sein duquel des possibilités inattendues peuvent se dessiner. En classe, comme c'est le cas dans les pratiques professionnelles, le remue-méninges doit autoriser les élèves à émettre librement leurs idées (même les plus farfelues) en suspendant les jugements. En outre, le PCT est un processus réfléchi qui implique une génération systématique et intelligente de concepts, et par lequel il s'agit de produire des solutions hypothétiques plausibles (Dym et al., 2005). En conséquence, la conception technique ne doit pas reposer uniquement sur des " éclairs de génie » comme le soulignent Dym et al., 2005 (p. 104) : "While creativity is important, and may even be teachable, design is not invention as caricatured by the shouting of "Eureka" and the flashing of a light bulb. ». Ainsi, en classe, le remue-méninges ne doit pas être envisagé comme une activité de recueil de conceptions sans préparation préalable. Il doit être source d'inspiration. C'est pourquoi la

10 On distingue essentiellement deux types de fonctions : 1) La fonction principale ou globale qui permet de décrire la finalité de l'objet technique et qui répond à la question : à qui et à quoi l'objet technique sert-il ? ; 2) Les fonctions techniques qui traduisent la partie rationnelle du besoin et qui sont internes à l'objet technique. Les fonctions techniques impliquent une réflexion sur des solutions techniques et répondent à la question : par quelles actions l'objet technique peut-il rendre service?

11 Les contraintes expriment les limitations dans la conception de l'objet technique. Elles peuvent être liées à des aspects techniques, ergonomiques, réglementaires, budgétaires, esthétiques, sécuritaires, etc.

12 "Asking questions emerges as a beginning step of any design project or class in the problem definition phase " (Dym et al., 2005, p. 104). 
génération de solutions créatives s'accompagne généralement d'une recherche (dans des sources variées) et d'une analyse de l'existant (Davis et al., 2002; Mentzer, 2011b). À cette étape, le recours à des analogies peut s'avérer utile pour ouvrir ou restreindre l'espace de recherche d'idées ou de sources d'inspiration (Bonnardel, 2009). Dès lors, il s'agit pour l'enseignant.e de solliciter les élèves à explorer, comparer et analyser des principes utilisés dans des objets techniques existants qui puissent être mobilisés ou adaptés à l'objet technique à construire. Comme toutes les solutions ne sont pas de valeur égale, il y a nécessité de procéder à la sélection d'une solution en recourant à un processus d'optimisation sur la base d'explorations documentaires ou empiriques (Hynes et al., 2011). La génération et la sélection des solutions créatives impliquent par ailleurs la gestion de différentes contraintes; celles-ci interviennent de façon déterminante dans la définition (et la redéfinition) du problème de conception et dans sa résolution, comme le relève Bonnardel (2009, p. 12) qui stipule :

Pour que la résolution du problème de conception soit efficace, le concepteur doit non seulement rechercher de la nouveauté (et, pour cela, élargir, dans un premier temps, son espace de recherche), mais il doit aussi parvenir à une solution adaptée aux contraintes de la situation (et donc restreindre progressivement son espace de recherche). Dans ce but, le concepteur se construit une représentation mentale intégrant des contraintes variées $[\ldots]$.

Contrairement aux novices, les ingénieur.e.s expérimenté.e·s passent plus de temps à juger de la faisabilité d'une solution en s'appuyant rigoureusement sur les éléments qui fondent le problème : les contraintes, les critères et les fonctions (Mentzer et al., 2015). Enfin, soulignons que la génération et la sélection de solutions en vue de produire une solution optimisée impliquent une approche itérative. C'est la nature même du PCT qui consiste selon Dym et al. (2005, p. 104) en " an iterative loop of divergent-convergent thinking ». Selon les cas de figure, une ou plusieurs phases du PCT peuvent être réitérées. Ainsi, dans un PCT, un cycle d'élaboration et d'évaluation de solutions créatives permet au concepteur ou à la conceptrice de décider de développer la solution courante, de la redéfinir partiellement ou d'en rechercher une autre en réitérant le cycle d'élaboration-évaluation (Bonnardel, 1999). Ainsi, le PCT ne doit pas être envisagé comme un processus linéaire où il agit de suivre, selon un ordre chronologique, un ensemble d'étapes sans faire de retour en arrière. Des retours en arrière, des remises en cause de décisions antérieures ou encore des reports à plus tard d'autres décisions sont choses fréquentes (Bonnardel, 1999). La mise en œuvre d'un premier cycle de conception permet d'aboutir à un prototype, lequel fait l'objet d'améliorations jusqu'à l'obtention d'un produit final qui répond de manière plus satisfaisante aux exigences du problème posé. Dans bien des cas, il s'agit de reprendre certaines phases du PCT (Adams et Atman, 2000 ; Atman et al., 1999). Par exemple, après avoir conçu un prototype, un·e ingénieur.e peut refaire un remue-méninges avec ses collègues ou se documenter de manière plus approfondie pour améliorer un principe technique qui n’est pas tout à fait au point avant d'aller plus loin dans l'élaboration d'une solution finale.

\subsection{Analyser et modéliser}

Dans tout PCT, pour développer et réduire le champ des possibles, les ingénieur.e.s recourent à des analyses prédictives et des approches de modélisation (Grubbs, 2016 ; Smith, 2006). L'analyse peut porter sur diverses dimensions du problème (ex. : écologique, financière, technique, etc.) (Mentzer, 2011b). Quant à la modélisation, elle est une tâche sous-jacente à la génération des solutions, car elle implique le recours à des modèles mathématiques ou analytiques pour exprimer certains aspects de l'objet technique (ou de ses composantes) au regard des fonctions (globale ou techniques) (Dym et al., 2005). Mais selon Dugger (1994, p. 7), à l'école, le PCT est " guided by trial and error or skilled approaches derived from the concrete " alors que chez les ingénieur.e.s, il est plutôt " guided by a more analytical study with specific solutions recommended ». Par conséquent, les approches de modélisation scolaires ne devraient pas nécessiter le recours à des modèles mathématiques très sophistiqués.

Lorsqu'on parle de modélisation, il est nécessaire de distinguer entre la modélisation cognitive ${ }^{13}$ (processus d'élaboration des modèles mentaux) (Johnson-Laird, 1983) et la modélisation pratique et concrète (processus d'élaboration des modèles matériels ou matérialisation des modèles mentaux). Dans tout PCT, l'une ne va pas sans l'autre comme le soulignent Kimbell et al. (1991). Ces auteurs considèrent le PCT comme une activité de modélisation impliquant une interaction dynamique entre les idées produites par la pensée et les idées concrétisées dans la réalité (figure 2) : "It is our contention that this inter-relationship between modelling ideas in

13 À la suite de Parkinson (2007), nous désignons la modélisation cognitive en ingénierie comme étant celle qui permet de produire des idées de conception au niveau de la pensée. 
the mind, and modelling ideas in reality is the cornerstone of capability in design and technology. It is best described as "thought in action" " (Kimbell et al., 1991, p. 21).

\section{Figure 2}

Le PCT comme modélisation d'une pensée en action (Kimbell et al.,1991, p. 20)

THE INTERACTION OF MIND AND HAND

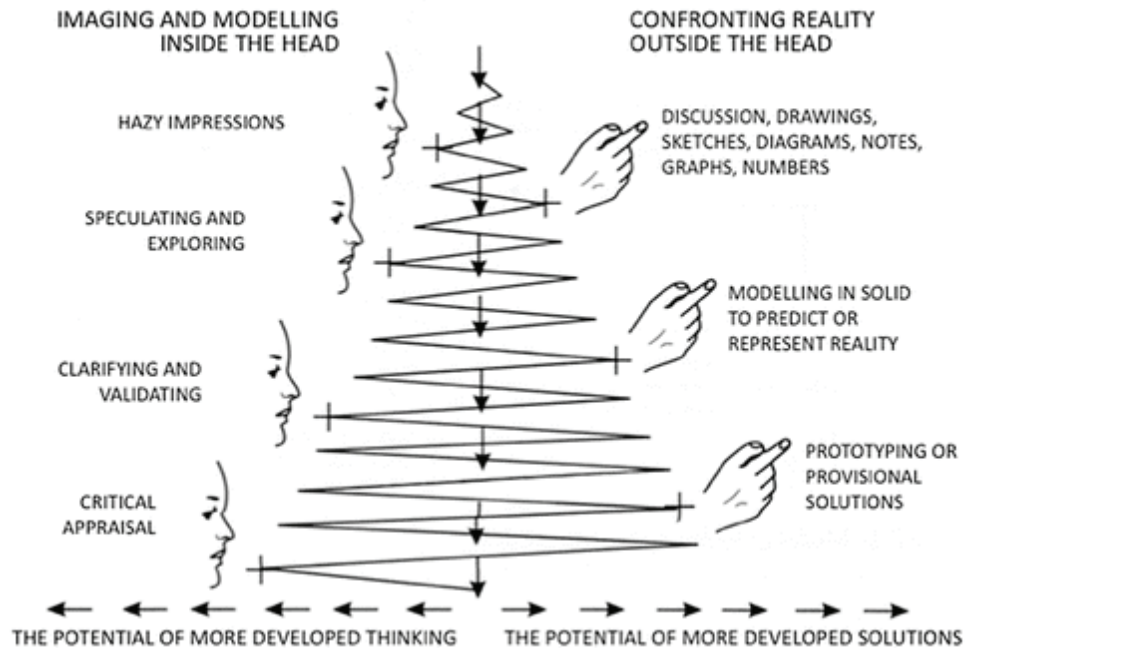

L'idée d'envisager le PCT comme la modélisation d'une pensée en action met en exergue que la modélisation ne se réduit ni à une activité cognitive (du monde conceptuel) ni à une activité matérielle (du monde empirique), mais qu'elle consiste plutôt en une activité permettant d'articuler les deux mondes de manière dynamique. Ainsi, les tâches que les élèves peuvent faire dans le monde matériel (monde des matériaux, des objets, etc.) ne se réalisent pas selon un processus aléatoire ou indéterminé. Elles sont guidées par un schéma conceptuel fondé sur l'expérimentation dans les limites des phonèmes physiques (Parkinson, 2007). De même, les idées de conception ("design ideas") que les élèves peuvent produire dans le monde conceptuel ne doivent pas rester uniquement sous la forme de conceptualisations abstraites (Parkinson, 2007, p. 242) :

Design ideas may have a variety of sources. They may be inspirations ; flashes of imagination given some mental form. Design ideas may be modifications or adaptations of existing things. Design ideas may be specifications or possibilities for action. The key term here is that relating to the nature of "possibility".

En des mots plus simples, les idées de conception doivent être matérialisées dans les artéfacts construits par les élèves. La modélisation joue un rôle central dans le développement de la pensée design (Bonnardel, 2006, 2009 ; Didier, 2017). Cette forme de pensée spécifique (appelée aussi la pensée technique) que certains philosophes de la technique (Combarnous, 1984 ; Deforge, 1981 ; Séris, 1994) associent à la technicité mobilise l'habileté à recourir au graphisme technique ${ }^{14}$ (Deforge, 1981 ; Rabardel et Weill-Fassina, 1987), lequel peut être appréhendé comme "support de la création ou de la recherche depuis les premiers croquis fonctionnels jusqu'aux dessins achevés " (Roy et Bousadra, 2017, p. 17). Les modèles en technologie sont très diversifiés (ex. : modèles littéraires, modèles graphiques, modèles informatiques, etc.) et ont plusieurs fonctions (ex. : s'informer, expérimenter, évaluer, communiquer, etc.) selon les intentions du modélisateur ou de la modélisatrice (Parkinson, 2007). Les modèles graphiques, en particulier les croquis et les schémas, font l'objet d'une grande utilisation chez ingénieur.e.s, mais aussi dans les pratiques de classe.

Le croquis est un dessin réalisé à main levée (sans l'aide d'instruments de dessin), pour représenter sommairement un objet sans recherche de détails. Dans la phase initiale d'un projet de conception, le croquis est fréquemment utilisé par les ingénieur·e.s en raison de ses nombreuses fonctions (Do et al., 2000 ; Dym et al.,

14 Le graphisme technique est un langage graphique figuratif normalisé par des conventions pour l'analyse, la conception et la communication des objets techniques. Il est utilisé principalement pour la représentation des composantes structurelles et du fonctionnement de ces objets dans plusieurs disciplines des sciences de l'ingénierie, en particulier en génie mécanique, en génie électrique et en génie civil. 
2005 ; Schütze et al., 2003) : exprimer une première idée ou un concept, développer, réviser et affiner des idées, faciliter l'analyse et la communication, améliorer la qualité des solutions produites, etc. Les croquis font partie du monde des inventions. Le dessin de brevet pour la machine volante de R. J. Spalding (3 mai 1889) est un magnifique croquis emblématique (figure 3) ${ }^{15}$.

\section{Figure 3}

Dessin de brevet pour la machine volante de R. J. Spalding (3 mai 1889)

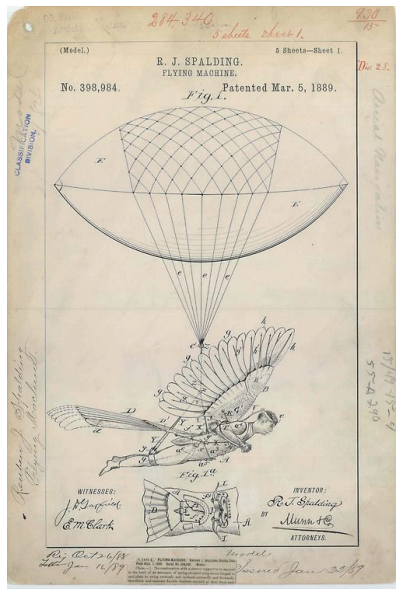

Plus récemment, sur le plan historique, la figure $4{ }^{16}$ présente deux croquis à main levée d'un système de freinage d'une bicyclette avec étriers à tirage central, l'ancêtre de tous les freins sur jantes modernes inventé par le Français Mafac. À la demande de l'enseignant·e, un.e élève peut être appelé.e à produire de tels croquis et à les accompagner d'une description écrite ou verbale. En utilisant le langage technique, il ou elle décrira ce système qui comporte deux mâchoires ressemblant à des ciseaux recourbés et qui est fixé à l'avant et l'arrière du vélo sur un pivot central (1). En tirant sur les bouts supérieurs de ces mâchoires, ces dernières se referment et pressent les patins sur la jante. Les mâchoires sont reliées par un câble d'acier appelé le cavalier (2), lequel est fixé aux étriers par une boule de métal (4) à un bout. Quand on actionne le levier de frein, on tire sur le cavalier grâce à un petit support (3) et on contracte les mâchoires. Le câble d'acier est fixé en général sous la potence (5).

\section{Figure 4}

Croquis d'un système de freinage d'une bicyclette avec étriers à tirage central
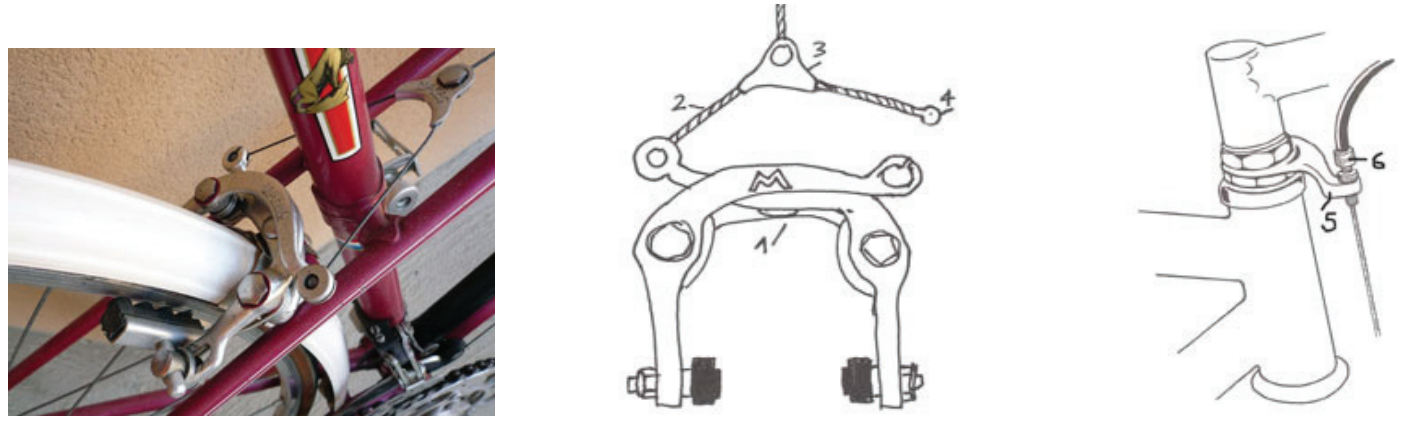

Le schéma est une représentation iconique un peu plus détaillée que le croquis. Il est réalisé au moyen de symboles normalisés. On peut distinguer entre plusieurs types de schémas, parmi lesquels le schéma de construction, le schéma de principes et le schéma de fonctions.

Le schéma de principes (ou de fonctionnement) est un dessin simplifié qui représente par des symboles normalisés le fonctionnement d'un objet technique. Il ne doit préciser que les résultats souhaités et les principaux principes

15 Source : https://www.pinterest.com/pin/313281717823201307/.
16 Source: http://mapage.noos.fr/ptis.trucs.sympas/freins_velo.htm. 
de fonctionnement. On schématise généralement les pièces qui entrent en jeu dans le fonctionnement de l'objet technique, les forces d'action qui permettent à l'objet de fonctionner, les organes d'entrée (ceux qui reçoivent une action de l'environnement), les organes de sortie (ceux qui exercent une action sur l'environnement), les organes intermédiaires (organes principaux) et les mouvements des pièces qui sont engendrés par ces forces. Le schéma de principes peut être évolutif, c'est-à-dire qu'au fur et à mesure que la conception de l'objet évolue, le schéma initial se transforme et comporte des éléments ou des principes additionnels. Le schéma final présente alors les principes retenus. La figure $5^{17}$ présente un schéma de principes d'un arrêt de porte.

\section{Figure 5}

Schéma de principes d'un arrêt de porte
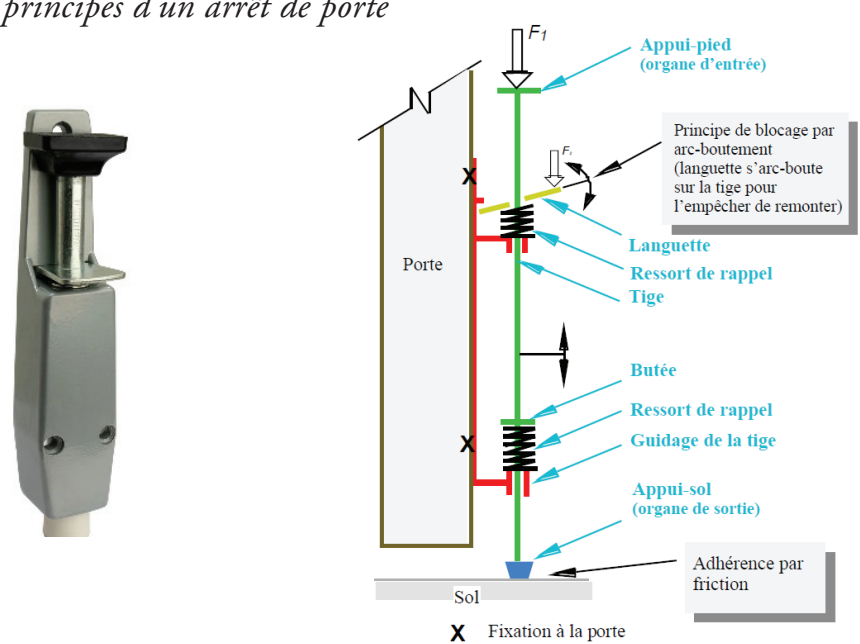

Le schéma de construction (ou schéma constructif) est utilisé pour représenter les solutions techniques adoptées pour solutionner les problèmes soulevés dans le schéma de principes. On schématise généralement les organes concernant directement le fonctionnement (organes de guidage, de transmission, etc.), les organes mobiles (organes de réglage, de blocage, etc.), les organes de liaison ou le symbole du caractère de la liaison réalisée et les organes intermédiaires. Les noms des pièces et des matériaux entrant dans sa fabrication peuvent être aussi spécifiés. La figure $6^{18}$ présente de schéma de construction d'un arrêt de porte.

\section{Figure 6}

Schéma de construction d'un arrêt de porte
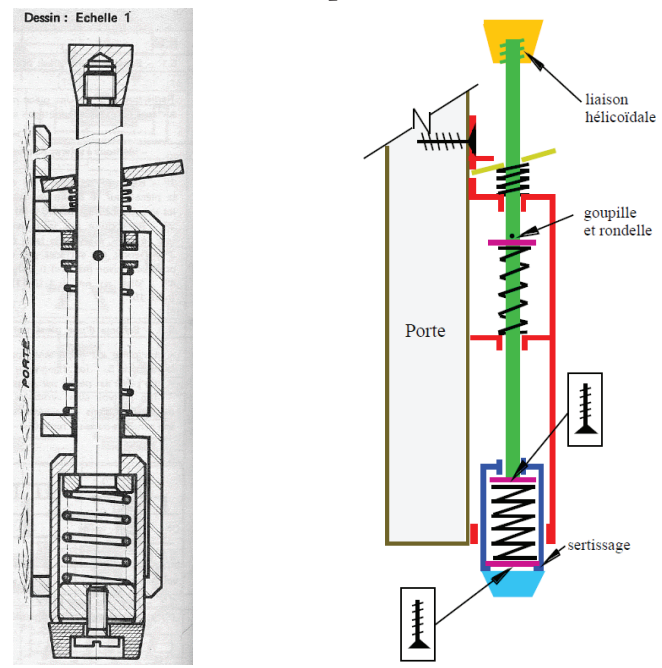

17 Source: http://cdpsciencetechno.org/wp-content/uploads/2013/11/schematisation1.pdf.

18 Source : http://cdpsciencetechno.org/wp-content/uploads/2013/11/schematisation1.pdf. 
Quant au schéma de fonctions, il est utilisé pour faire l'analyse fonctionnelle d'un objet technique en le décomposant en un ensemble de sous-systèmes. Il se présente comme un diagramme mettant en valeur les fonctions et les solutions techniques envisagées. Ce schéma est désigné sous le vocable du Functional Analysis System Technique (FAST) par les ingénieur.e.s. Il consiste à réaliser, à partir d'une fonction principale à satisfaire, une décomposition en fonctions techniques (pour les sous-systèmes de cet objet) pour aboutir à des solutions techniques. La figure 7 présente un exemple de schéma de fonctions d'une machine à café pour la fonction principale qui consiste à préparer les boissons.

\section{Figure 7}

Schéma de fonctions d'une machine à café filtre pour la fonction principale "Préparer le café "

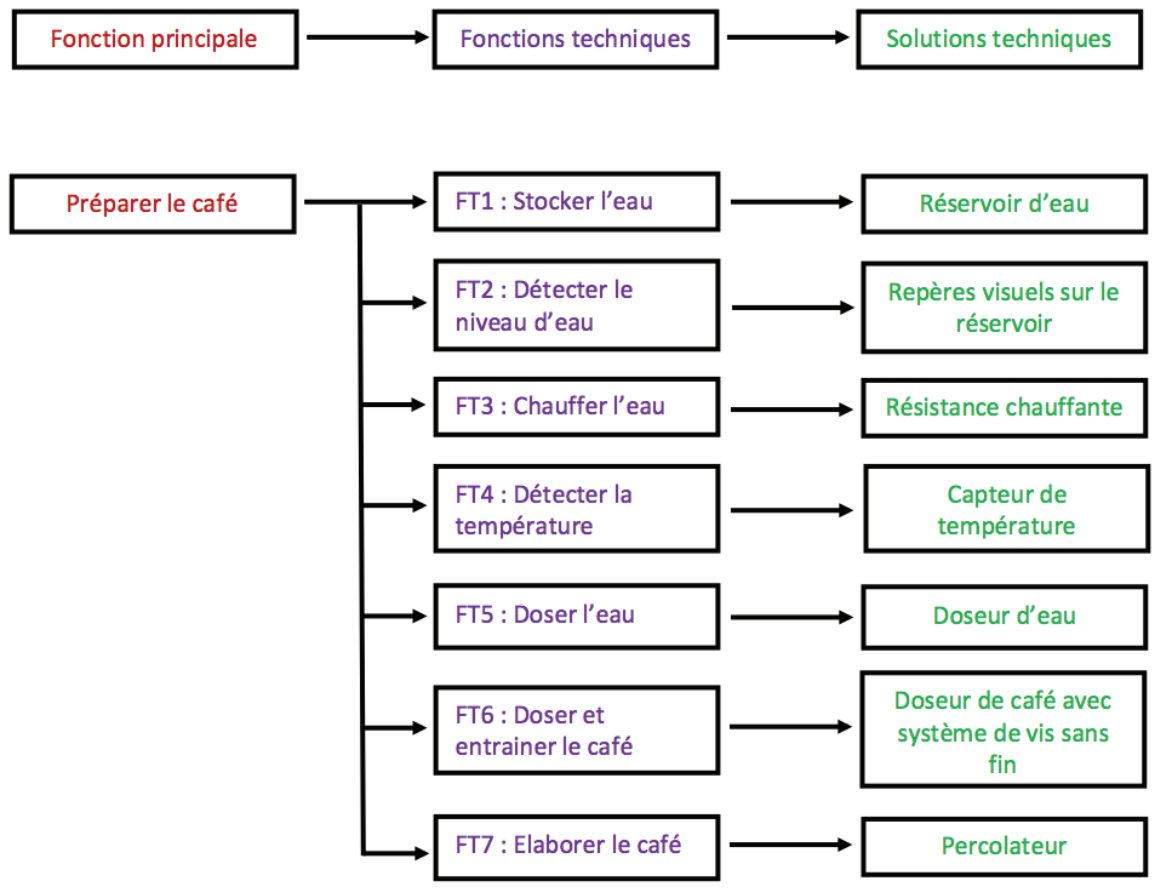

\subsection{Expérimenter et évaluer}

La génération des solutions créatives est associée à une étape dite d'évaluation, de vérification ou de validation (Bonnardel, 2009). L'expérimentation est guidée par l'analyse et la modélisation dans le but de valider les prototypes, et de fournir des preuves empiriques lorsque les données sont insuffisantes : "The design of systems is rarely accomplished exclusively by applying fundamental scientific principles. In most cases, the design of systems also requires some use of empirical data and experimentation » (Dym et al., 2005, p. 106). Elle consiste à mettre à l'épreuve le prototype. L'interrelation entre l'expérimentation et la modélisation permet de guider le PCT. Quant à l'évaluation, cette tâche est également guidée par l'analyse et la modélisation et nécessite un retour sur la définition du problème. En effet, celle-ci survient lorsque "we compare our analyses of the attributes and behavior of the current design to the stated design specifications and constraints to see if this synthesis is acceptable " (Dym, 1994, p. 28). En d'autres mots, elle permet de remettre en question la pertinence des solutions hypothétiques avancées au fur et à mesure que progresse le PCT, et d'identifier une solution optimisée au terme de ce processus (Smith, 2006). Si lors de la génération des solutions, c'est essentiellement la composante pensée divergente de la pensée design qui est activée pour produire des idées créatives, lors de l'expérimentation et l'évaluation, c'est plutôt la composante pensée convergente ${ }^{19}$ qui l'est (Dym et al., 2005). L'évaluation du prototype peut se faire selon deux modalités : au moyen d'une évaluation théorique qui consiste à porter

19 Selon Dym et al. (2005, p. 104), " effective inquiry in design thinking includes both a convergent component of building up to asking deep reasoning questions by systematically asking lower-level, convergent questions, and a divergent component in which generative design questions are asked to create the concepts on which the convergent component can act ». 
un regard critique sur l'adéquation de l'objet technique par rapport aux fonctions, contraintes et spécifications indiquées dans le cahier des charges fonctionnel ou au moyen d'une évaluation empirique qui consiste à porter un regard critique sur le fonctionnement de cet objet lors de son usage en atelier ou dans la vie quotidienne. Afin que l'évaluation d'un prototype s'appuie sur l'analyse et la modélisation, nous proposons de reprendre les outils de modélisation qui ont guidé la conception et la fabrication de celui-ci. Dès lors, la qualité du prototype peut être évaluée à l'aune des questions suivantes : est-ce que le prototype permet de répondre au problème posé ? Le fonctionnement du prototype est-il satisfaisant pour les conditions d'usage prévues ? Est-ce que le prototype satisfait le besoin exprimé initialement au regard de sa fonction globale ? Est-ce que les solutions techniques proposées pour chacune des fonctions techniques spécifiques sont satisfaisantes ? Pour remplir une même fonction technique, est-ce qu'une autre solution technique serait plus efficace ? Les objectifs de performance sont-ils atteints? Les contraintes sont-elles respectées? Quelles sont les limites du prototype ? Sur quels aspects celui-ci pourrait-il être amélioré ? etc.

\subsection{Communiquer}

Presque toutes les tâches en ingénierie sont des tâches sociales et communicatives réalisées par des équipes souvent pluridisciplinaires. Les PCT efficaces exigent de la part des ingénieur.e.s qu'ils ou elles soient capables de penser et de communiquer à des publics hétérogènes (autres ingénieur.e.s, client.e.s, techniciens et techniciennes, administrateurs et administratrices, etc.) tout en mobilisant des langages et des registres de représentations sémiotiques variés (Dym et al., 2005 ; Trevelyan, 2010 ; Vinck, 2003). La communication opère de manière transversale dans toutes les phases d'un PCT. La PEI analyser et modéliser met bien en évidence sont lien étroit avec la PEI communiquer. Elle se produit au moyen de différents langages et selon plusieurs finalités (Dym et al., 2005, p. 108) : «Different languages are employed to represent engineering and design knowledge at different times, and the same knowledge is often cast into different forms or languages to serve different purposes. ». Il apparait que les concepteurs ou conceptrices expérimenté·e·s, contrairement aux novices, utilisent une plus grande diversité de langages et de registres de représentations sémiotiques pour communiquer leurs idées (Crismond et Adams, 2012, p. 758) telles que les " gestures, words, and artifacts, make drawings, construct physical prototypes, and create virtual models ». Dym et al. (2005, p. 108) énoncent six types de langages utilisés dans les PCT, tant dans la pratique que dans la recherche, lesquels sont associés à des finalités spécifiques (tableau 4) (en annexe). L'exercice de l'activité d'ingénierie exige la maitrise de ces langages.

Comme c'est le cas dans le pratiques professionnelles, la mise en ouvre d'un PCT en classe implique souvent la coordination simultanée de plusieurs langages et registres de représentations sémiotiques (Dym et al., 2005 ; Kelly et Cunningham, 2019). Dans un PCT, les élèves communiquent lorsque, par exemple, ils complètent un cahier des charges fonctionnel avec l'aide de l'enseignant.e, décrivent les principales fonctions techniques d'un objet technique, modélisent la structure ou le fonctionnement d'un objet technique au moyen de modèles graphiques appropriés, échangent leur compréhension sur un principe technique proposé dans un ouvrage de référence, argumentent sur la pertinence de l'utilisation d'une solution technique originale pour satisfaire une fonction technique, expliquent les écarts entre le pensé (modèle) et le réalisé (solution finale), débattent sur le choix d'un prototype parmi plusieurs au regard d'un ensemble de contraintes, portent un regard critique sur le PCT mis en œuvre et identifient des pistes d'amélioration possibles.

\subsection{Prendre des décisions}

Les ingénieur.e.s sont appelé.e.s à prendre des décisions (Dym et al., 2005, p. 107) dans les PCT qu'ils conduisent : "Design is a rational process of choosing among alternatives ». Plusieurs méthodes peuvent être envisagées pour soutenir cette tâche. La prise de décision s'inscrit dans un processus interdisciplinaire, en ce sens que les décisions en matière de conception sont souvent orientées sur la base de fondements scientifiques ou mathématiquement. Hazelrigg (1994) (cité dans Dym et al. 2005, p. 107) a fait valoir que pour conduire un PCT rationnel qui permet de produire « the best possible results..., a mathematics of design is needed... based on the recognition that engineering design is a decision-intensive process and adapting theories from other fields such as economics and decision theory ». En classe, lors de la construction d'un objet technique, de telles décisions sont également prises sur la base de fondements scientifiques. À titre d'exemple, lors de la conception d'un véhicule miniaturisé disposant d'un dispositif de propulsion autonome, on améliorera la puissance de ce véhicule si l'on réduit sa masse (ce qui réduira le poids lié à l'effet de la gravité) en utilisant un châssis composé de matériaux légers. Lorsque des ingénieur·e·s expérimenté·e·s doivent effectuer une analyse comparative de plusieurs prototypes en vue d'optimiser le couple besoin-solution, dans le cas où de nombreuses contraintes sont en jeu et qu'elles n'ont pas toutes la même valeur, ils ou elles utilisent une matrice de décisions avec un système de pondération des contraintes. Les décisions relatives à la qualité d'une conception technique impli- 
quent une implication active des membres de l'équipe et la construction d'une solution optimisée basée sur la rationalité (Davis et al., 2002). En classe : «A decision matrix helps assist students in objectively considering the alternatives based on their advantages and disadvantages " (Gomez et al., 2004, cité dans Mentzer, 2011b). À titre d'exemple, Draper (2009) présente dans son article une matrice de décisions ayant servi à sélectionner la première parmi trois idées de conception d'un produit en fonction de trois contraintes (coût, sécurité et facilité d'utilisation) pondérées différemment selon leur degré d'importance (figure 8). L'utilisation d'un tel outil en classe peut permettre de mettre en exergue que l'évaluation n'est pas un processus subjectif, mais rationnel.

\section{Figure 8}

Matrice de décision (Draper, 2009, p. 9)

\begin{tabular}{|l|l|l|l|l|}
\hline Criteria & Importance \% & Idea \#1 & Idea \#2 & Idea \#3 \\
\hline \multirow{2}{*}{ Cost } & & 10 & 5 & 4 \\
\hline & $30 \%$ & $10 \times 30=300$ & $5 \times 30=150$ & $4 \times 30=120$ \\
\hline Safety & $20 \%$ & 4 & 9 & 9 \\
\hline & & $5 \times 20=80$ & $9 \times 20=150$ & $9 \times 20=180$ \\
Easy to Use & $50 \%$ & $5 \times 50=250$ & $6 \times 50=300$ & 3 \\
\hline
\end{tabular}

\section{Conclusion et discussion}

Dans cet article, nous avons développé un répertoire de six PEI qu'il peut être pertinent de faire acquérir aux élèves dans le cadre d'un enseignement de la technologie centré sur le PCT : définir un problème technique, générer et sélectionner des solutions en recourant à une approche systématique et itérative, analyser et modéliser, expérimenter et évaluer, communiquer et prendre des décisions. Les PEI sont des pratiques discursives interdisciplinaires épistémologiquement cohérentes avec les pratiques sociotechniques de référence (celles des ingénieur·e.s) et caractérisent la pensée design des élèves. Elles participent pleinement à la construction d'une Communauté Discursive Interdisciplinaire Scolaire où le développement d'une culture technique repose sur une acculturation des élèves à des manières de penser, de parler et d'agir spécifiques (Bernié, 2002 ; Jaubert et al., 2004) de la technologie appréhendée dans une perspective interdisciplinaire. L'apprentissage de la langue de la technologie permet aux élèves de construire un rapport au monde différent de celui que peuvent offrir les sciences de la nature. La langue des sciences n'est pas adaptée pour définir et résoudre un problème technique. S'engager dans un PCT, ce n'est pas comme s'engager dans une investigation scientifique. Ces processus sont épistémologiquement et méthodologiquement différents. Ils se distinguent quant à leurs finalités, la nature de leurs problèmes, leurs modalités d'enquête, leurs savoirs mobilisés et leurs solutions. De notre point de vue, l'une des missions de l'enseignant.e est de faire apprendre à ses élèves le vocabulaire spécifique de la technologie. Sur le plan curriculaire, cela devrait se traduire par une mise en texte permettant de distinguer clairement les finalités, les contenus et les modalités opératoires de la technologie de celles des autres disciplines, en particulier les sciences de la nature. Or, le Plan d'études romand (PER) (CIIP, 2010), pour la scolarité primaire (élèves âgé.e.s de 6 à 12 ans), propose une mise en texte holistique de la technologie où celle-ci est fusionnée avec les sciences de la nature. Le PCT est présenté comme un processus caractéristique des sciences expérimentales et apparait de manière parcellaire à travers les énoncés de texte, ce qui peut suggérer aux enseignant·e·s que le PCT constitue un cas particulier de la démarche scientifique, et provoquer ainsi un brouillage épistémologique important.

La culture technique devrait être considérée comme une composante fondamentale des curricula de toute la scolarité obligatoire. Pourtant, dans plusieurs systèmes éducatifs, la culture littéraire, mathématique ou scientifique est encore bien souvent privilégiée à la culture technique. Des philosophes de la technique (Haudricourt, 1987 ; Roqueplo, 1983 ; Simondon, 1958) ont déjà signifié que l'absence d'une culture technique dans la population en général figure parmi les plus grandes causes d'aliénation du monde contemporain. Ce désintéressement de l'humain envers la culture technique n'est pas nouveau. Dans leur célèbre Encyclopédie, Alembert et Diderot relevaient, il y a plus de deux siècles, qui si les techniques font partie intégrante du patrimoine culturel de l'humanité, la tendance générale est de faire des éloges aux inventions scientifiques, mais de mépriser les arts mécaniques. Pour contrer cette aliénation généralisée au monde technique, Simondon (1958) proposait 
de redonner à la culture générale le caractère véritable qu'elle a perdu en réintroduisant en elle la conscience de la nature des machines, de leurs relations mutuelles et de leurs relations avec l'humain. Il affirmait " qu'une initiation aux techniques doit être placée sur le même plan que l'éducation scientifique " (Simondon, 1958, p. 13). Qu'en est-il dans notre société actuelle ?

Il apparait que la technologie est souvent prise pour un concept " fourretout " et polysémique. Elle est souvent associée par plusieurs aux technologies de l'information, à l'utilisation d'outils et de machines sophistiquées, ou encore à une activité exclusivement réservée aux spécialistes (Käser, 2017). À ce propos, nous aimerions faire une remarque importante. La technologie qui se fait à l'école ne doit ni être confondue avec la technique, ni se positionner de manière surplombante à celle-ci, car « dans le concret, dans la vie réelle, c'est l'activité technique qui précède, engendre et donc prouve l'existence de la technicité (aptitude de l'humain), des techniques (somme d'expériences accumulées) et de la technologie (étude des techniques) " (Combarnous, 1984, p. 35). Dans le même sens, Séris (1994, p. 1-3) relève que « la technologie ne doit pas nous cacher les techniques. Elle devrait même nous aider à mieux les comprendre. [...] L'attention aux techniques, et donc au technique, n’a jamais été aussi envahissante que dans nos sociétés post-industrielles ». Ces propos légitiment le choix d'opter pour un enseignement de la technologie centré sur le PCT.

Si la préoccupation est partagée par plusieurs sur la nécessité de faire ce type d'enseignement, celui-ci poserait néanmoins des défis importants aux enseignant.e.s. En effet, lorsque des activités techniques sont mises en œuvre en classe, elles se réduisent souvent à des activités de bricolage ou des activités scientifiques déguisées non problématisées dont les tâches sont épistémologiquement incohérentes avec les pratiques sociotechniques de référence (celles des ingénieur.e.s) (Bousadra, 2014 ; Bousadra et Hasni, 2012 ; Lebeaume, 2012). De nombreux obstacles peuvent empêcher d'introduire avec succès le PCT à l'école obligatoire comme la faible promotion de l'enseignement de l'ingénierie par les autorités scolaires, la surcharge des plans d'études ou encore l'absence d'orientations claires au sujet de l'ingénierie dans les curricula. Mais l'obstacle majeur serait l'absence d'une formation initiale ou continue adéquate des enseignant.e.s dans ce domaine de connaissance spécifique (Bequette et Bequette, 2012 ; Felix et al., 2010). Dans plusieurs institutions de formation des enseignant.e.s, le développement des compétences professionnelles en enseignement des mathématiques et des sciences est souvent privilégié à celui de la technologie (Borko, 2004 ; Van Driel et al., 2001 ; Walker, 2007). Qu'en est-il dans les Hautes écoles pédagogiques en Suisse?

Plusieurs travaux montrent que les enseignant.e.s de l'école obligatoire disposent souvent d'un faible sentiment de compétence personnelle au regard de la technologie, et n'ont ni les connaissances ni l'expérience nécessaires pour engager leurs élèves dans des PCT (Brophy et al., 2008 ; Capobianco et al., 2011 ; Cunningham et Hester, 2007 ; English et King, 2015 ; Kelley et Wicklein, 2009 ; Rohaan et al., 2012 ; Sanders, 2008). Plusieurs types de connaissances chez les enseignant.e.s ont une influence sur les apprentissages et les attitudes des élèves en technologie, en particulier la Subject Matter Knowledge (SMK) et la Pedagogical Content Knowledge (PCK) (Rohaan et al., 2010, 2011, 2012). Rohaan et al. (2012) ont montré que l'acquisition de la SMK (connaissance des concepts et des processus de la technologie, en particulier celle du PCT) chez des enseignant.e.s du primaire constitue une condition nécessaire et préalable à l'acquisition de la PCK (connaissance des conceptions des élèves, des approches et stratégies d'enseignement de la technologie), et que cette acquisition renforce en même temps leur sentiment de compétence personnelle dans ce domaine. Ces résultats mettent en évidence l'importance d'assurer une solide formation initiale et continue des enseignant.e.s dans ce domaine de connaissance. En vue de l'élaboration des futurs plans d'études en Suisse romande, nous nous permettons de formuler trois recommandations : 1) accorder une plus grande place à la discipline technologie pour tous les degrés de la scolarité obligatoire, cette discipline étant curieusement absente au cycle 3 (début de l'école secondaire) ; 2) revoir les finalités et les modalités opératoires de cette discipline de manière à ce qu'elles s'inscrivent en plus grande cohérence avec les pratiques sociotechniques de référence des ingénieur.e.s ; 3) valoriser un enseignement du PCT basé sur acquisition progressive des PEI, de l'école primaire à l'école secondaire. Si un jour une telle réorientation est opérée, nous pensons que le développement d'une culture technique authentique chez les élèves sera renforcé. 


\section{Références}

Adams, R. S. et Atman, C. J. (2000, jour mois). Characterizing engineering student design processes: An illustration of iteration [communication orale]. Annual conference proceedings of the American Society for Engineering Education, session 2330, St Louis, MO.

Aguirre-Muñoz, Z. et Pantoya, M. L. (2016). Engineering literacy and engagement in kindergarten classrooms. Journal of Engineering Education, 105(4), 630-654.

Astolfi, J.-P. (2008). La saveur des savoirs : disciplines et plaisir d'apprendre. ESF.

Atman, C. J., Adams, R. S., Cardella, M. E., Turns, J., Mosborg, S. et Saleem, J. (2007). Engineering design processes : A comparison of students and expert practitioners. Journal of engineering education, 96(4), 359-379.

Atman, C. J., Chimka, J. R., Bursic, K. M. et Nachtmann, H. L. (1999). A comparison of freshman and senior engineering design processes. Design studies, 20(2), 131-152.

Bequette, J. W. et Bequette, M. B. (2012). A place for art and design education in the STEM conversation. Art education, 65(2), 40-47.

Berland, L., Steingut, R. et Ko, P. (2014). High school student perceptions of the utility of the engineering design process : Creating opportunities to engage in engineering practices and apply math and science content. Journal of Science Education and Technology, 23(6), 705-720.

Bernié, J.-P. (2002). L'approche des pratiques langagières scolaires à travers la notion de " communauté discursive " : un apport à la didactique comparée? Revue française de pédagogie, 141, 77-88.

Bogusch, L. L., Turns, J. et Atman, C. J. (2000). Engineering design factors : how broadly do students define problems ? 30th Annual Frontiers in Education Conference. Building on A Century of Progress in Engineering Education. Conference Proceedings (IEEE Cat. No. 00CH37135), 2, S3A-7. IEEE.

Bonnardel, N. (1999). L'évaluation réflexive dans la dynamique de l'activité du concepteur. Dans J. Perrin (Éd.), Pilotage des activités de coneception (pp. 87-105). L'Harmattan.

Bonnardel, N. (2006). Créativité et conception, approches cognitives et ergonomiques. Solal.

Bonnardel, N. (2009). Activités de conception et créativité : de l'analyse des facteurs cognitifs à l'assistance aux activités de conception créatives. Le travail humain, 72(1), 5-22.

Borillo, M. et Goulette, J.-P. (2002). Cognition et création : explorations cognitives des processus de conception. Mardaga.

Borko, H. (2004). Professional development and teacher learning : Mapping the terrain. Educational researcher, 33(8), 3-15.

Bousadra, F. (2014). L'enseignement par projets en sciences et technologies : études des pratiques d'enseignement chez des enseignants du secondaire au Québec [Thèse de doctorat]. Université de Sherbrooke.

Bousadra, F. et Hasni, A. (2012). Lapproche par projets et les savoirs disciplinaires en classe de sciences et technologies au Québec : compatibilité ou incompatibilité ? Présentation d'études de cas. Recherches en didactique, 13, 67-84.

Brophy, S., Klein, S., Portsmore, M. et Rogers, C. (2008). Advancing engineering education in P-12 classrooms. Journal of Engineering Education, 97(3), 369-387.

Burghardt, M. D. (1999). Introduction to engineering design and problem solving. McGraw Hill.

Capobianco, B. M., Diefes-dux, H. A., Mena, I. et Weller, J. (2011). What is an engineer ? Implications of elementary school student conceptions for engineering education. Journal of Engineering Education, 100(2), 304-328.

Capobianco, B. M., Yu, J. H. et French, B. F. (2015). Effects of Engineering Design-Based Science on Elementary School Science Students' Engineering Identity Development across Gender and Grade. Research in Science Education, 45(2), 275-292.

Conférence intercantonale de l'instruction publique de Suisse romande et du Tessin (CIIP). Plan d'études romand. En ligne : http://www.plandetudes.ch/home.

Combarnous, M. (1984). Comprendre les techniques et la technicité. Messidor/Ed. Sociales.

Crismond, D. P. et Adams, R. S. (2012). The informed design teaching and learning matrix. Journal of Engineering Education, $101(4), 738$.

Cunningham, C. M. et Hester, K. (2007, jour mois). Engineering is elementary : An engineering and technology curriculum for children [communication orale]. American Society for Engineering Education Annual Conference et Exposition, Honolulu, HI.

Cunningham, C. M. et Kelly, G. J. (2017a). Epistemic practices of engineering for education. Science Education, 101(3), 486-505.

Cunningham, C. M. et Kelly, G. J. (2017b). Framing engineering practices in elementary school classrooms. International Journal of Engineering Education, 33(1), 295-307.

Demailly, A. et Lemoigne, J.-L. (1986). Théories de la conception. Dans A. Demailly et J.-L. Lemoigne (dir.), Sciences de l'intelligence, sciences de l'artificiel (p. 435-446). PUL.

Daugherty, J. L. (2012). Infusing engineering concepts : Teaching engineering design. http://files.eric.ed.gov/fulltext/ED537384.pdf.

Davis, D. C., Gentili, K. L., Trevisan, M. S. et Calkins, D. E. (2002). Engineering design assessment processes and scoring scales for program improvement and accountability. Journal of Engineering Education, 91(2), 211-221.

Dearing, B. M. et Daugherty, M. K. (2004). Delivering engineering content in technology education. Technology and Engineering Teacher, 64(3), 8-11.

Deforge, Y. (1981). Le graphisme technique : son histoire et son enseignement (vol. 4). Éditions Champ Vallon.

Didier, J. (2017). Didactique de la conception et démocratie technique. Dans J. Didier, Y.-C. Lequin et D. Leuba (dir.), Devenir acteur dans une démocratie technique, Pour une didactique de la technologie (p. 137-152). Pôle éditorial de l'Université de Technologie Belfort-Montbéliard (UTBM).

Do, E. Y.-L., Gross, M. D., Neiman, B. et Zimring, C. (2000). Intentions in and relations among design drawings. Design studies, 21(5), 483-503.

Doppelt, Y., Mehalik, M. M., Schunn, C. D., Silk, E. et Krysinski, D. (2008). Engagement and achievements : A case study of design-based learning in a science context. Journal of technology education, 19(2), 22-39.

Dorst, K. (2011). The core of 'design thinking'and its application. Design studies, 32(6), 521-532.

Draper, S. (2009). Engineering design process. Technology and Children, 8-10. 
Dugger, W. E. (1994). The Relationship between Technology, Science, Engineering, and Mathematics. The Technology Teacher, 53(7), 5-23.

Dym, C. L. (1994). Engineering design : a synthesis of views. Cambridge University Press.

Dym, C. L., Agogino, A. M., Eris, O., Frey, D. D. et Leifer, L. J. (2005). Engineering design thinking, teaching, and learning. Journal of engineering education, 94(1), 103-120.

Eide, A. R., Jenison, R. D., Mashaw, L. H. et Northup, L. L. (2002). Introduction to engineering design and problem solving. McGraw-Hill.

English, L. D. et King, D. T. (2015). STEM learning through engineering design : fourth-grade students' investigations in aerospace. International Journal of STEM Education, 2(14), 1-18.

Ertmer, P. A., Stepich, D. A., Flanagan, S., Kocaman-Karoglu, A., Reiner, C., Reyes, L., Santone, A. L. et Ushigusa, S. (2009). Impact of guidance on the problem-solving efforts of instructional design novices. Performance Improvement Quarterly, 21(4), $117-132$.

Felix, A. L., Bandstra, J. Z. et Strosnider, W. H. (2010). Design-Based science for STEM student recruitment and teacher professional development. Mid-Atlantic ASEE Conference, Villanova University.

Fortus, D., Dershimer, R. C., Krajcik, J., Marx, R. W. et Mamlok-Naaman, R. (2004). Design-based science and student learning. Journal of Research in Science Teaching, 41(10), 1081-1110.

Fortus, D., Krajcik, J., Dershimer, R. C., Marx, R. W. et Mamlok-Naaman, R. (2005). Design-based science and real-world problem-solving. International Journal of Science Education, 27(7), 855-879.

Fourez, G. (1997). Qu'entendre par îlot de rationalité ? et par îlot interdisciplinaire de rationalité ? Aster, 1997, 25 (Enseignants et élèves face aux obstacles). http://documents.irevues.inist.fr/handle/2042/8686.

Fourez, G., Maingain, A. et Dufour, B. (2002). Approches didactiques de l'interdisciplinarité. De Boeck Université.

Gelb, M. (1996). Thinking for a change : Discovering the power to create, communicate and lead. Crown Books.

Grubbs, Michael E., Strimel, G. J. et Kim, E. (2018). Examining design cognition coding schemes for P-12 engineering/ technology education. International Journal of Technology and Design Education, 28(4), 899-920.

Grubbs, M. E. (2016). Further Characterization of High School Pre-and Non-Engineering Students' Cognitive Activity During Engineering Design [Thèse de doctorat]. Virginia Tech.

Hacioğlu, Y., Yamak, H. et Kavak, N. (2017). The opinions of prospective science teachers regarding STEM education : The engineering design based science education. Gazi Üniversitesi Gazi Eğitim Fakültesi Dergisi, 37(2), 649-684.

Hailey, C., Erekson, T., Becker, K. et Thomas, M. (2005). National center for engineering and technology education. The Technology Teacher, 64(5), 23.

Haudricourt, A. (1987). La technologie, science humaine. Recherches d'histoire et d'ethnologie des techniques. Éditions de la Maison des Sciences de l'Homme.

Hmelo, C. E., Holton, D. L. et Kolodner, J. L. (2000). Designing to learn about complex systems. The journal of the learning sciences, 9(3), 247-298.

Householder, D. L. et Hailey, C. E. (2012). Incorporating engineering design challenges into STEM courses. http://ncete.org/ flash/pdfs/NCETECaucusReport.pdf.

Hynes, M., Portsmore, M., Dare, E., Milto, E., Rogers, C., Hammer, D. et Carberry, A. (2011). Infusing engineering design into high school STEM courses. https://digitalcommons.usu.edu/cgi/viewcontent.cgi?article=1165\&context=ncete_publications

Jaubert, M., Rebière, M. et Bernié, J.-P. (2004). Significations et développement : quelles " communautés ». Dans C. H. Moro et R. Rickenmann (dir.), Situation éducative et significations (p. 85-104). De Boeck Université.

Johansson-Sköldberg, U., Woodilla, J. et Çetinkaya, M. (2013). Design thinking : past, present and possible futures. Creativity and innovation management, 22(2), 121-146.

Johnson-Laird, P. N. (1983). Mental Models : Towards a cognitive science of language, inference, and conciousness. Cambridge University Press.

Jonassen, D. H. (1997). Instructional design models for well-structured and III-structured problem-solving learning outcomes. Educational technology research and development, 45(1), 65-94.

Käser, A. (2017). Technik und Design/ Technique et Design Un nouvel outil didactique pour les activités créatrices et techniques. Dans J. Didier, Y. Lequin et D. Leuba (dir.), Devenir acteur dans une démocratie technique. Pour une didactique de la technologie (p. 121-146). UTBM.

Kelley, T. R. et Wicklein, R. C. (2009). Teacher Challenges to Implement Engineering Design in Secondary Technology Education. Journal of Industrial Teacher Education, 46(3), 34-50.

Kelly, G. J. (2016). Discourse practices in science learning and teaching. Dans S. Wortham, D. Kim et S. May (dir.), Encyclopedia of language and education, Vol. 3 : Discourse and education (p. 1-15). Springer.

Kelly, G. J., Chen, C. et Prothero, W. (2000). The epistemological framing of a discipline : Writing science in university oceanography. Journal of Research in Science Teaching: The Official Journal of the National Association for Research in Science Teaching, 37(7), 691-718.

Kelly, G. J. et Cunningham, C. M. (2019). Epistemic tools in engineering design for K-12 education. Science Education, 103(4), $1080-1111$.

Kelly, G. J., Cunningham, C. M. et Ricketts, A. (2017). Engaging in identity work through engineering practices in elementary classrooms. Linguistics and Education, 39, 48-59.

Kelly, G. J. et Green, J. (1998). The social nature of knowing: Toward a sociocultural perspective on conceptual change and knowledge construction. Dans B. Guzzetti et C. Hynd (dir.), Perspectives on conceptual change : Multiple ways to understand knowing and learning in a complex world (p. 145-181). Lawrence Erlbaum Associates.

Kelly, G. J. et Licona, P. (2018). Epistemic practices and science education. Dans M. Matthews (dir.), History, philosophy and science teaching (p. 139-165). Springer. 
Kimbell, R., Stables, K., Wheeler, T., Wosniak, A. et Kelly, V. (1991). The assessment of performance in design and technology. Schools Examinations and Assessments Council (SEAC).

Kolodner, J. L., Camp, P. J., Crismond, D., Fasse, B., Gray, J., Holbrook, J., Puntambekar, S. et Ryan, M. (2003). Problem-based learning meets case-based reasoning in the middle-school science classroom : Putting learning by design (tm) into practice. The journal of the learning sciences, 12(4), 495-547.

Lebeaume, J. (2001). Pratiques socio-techniques de référence, un concept pour l'intervention didactique : diffusion et appropriation par les enseignants de technologie. Dans A. Rouchier, G. Lemoyne et G. Mercier (dir.), Le génie didactique. Usages et mésusages des théories de l'enseignement (p. 127-142). De Boeck Université.

Lebeaume, J. (2012). La didactique de la technologie à la croisée des curriculums, des apprentissages et des enseignements disciplinaires, dans une perspective socio-historique générale. Dans M.-L. Elalouf, A. Robert et M.-F. Bishop (dir.), État des lieux et perspectives pour la recherche et la formation (p. 48-55). De Boeck Université.

Lewis, T. (2005). Coming to terms with engineering design as content. Journal of Technology Education, 16(2), 37-54.

Lindberg, T., Noweski, C. et Meinel, C. (2009). Design Thinking : Zur Entwicklung eines exporativen Forschungsansatzes zu einem überprofessioinellen Modell. Neuwerk-Zeitschrift für Designwissenschaft, 1, 47-53.

Martinand, J. L. (1982). Contribution à la caractérisation des objectifs de l'initiation aux sciences et techniques [Thèse d'État]. Université de Paris-Sud.

Martinand, J.-L. (2013). Questions pour la recherche : la référence et le possible dans les activités scientifiques scolaires. Recherches en didactiques, 2(16), 75-99.

Mehalik, M. M., Doppelt, Y. et Schuun, C. D. (2008). Middle-school science through design-based learning versus scripted inquiry : Better overall science concept learning and equity gap reduction. Journal of engineering education, 97(1), 71-85.

Mentzer, N. (2011a). Engineering design thinking and information gathering final report. http://ncete.org/flash/pdfs/Mentzer\%20 Final\%20Report.pdf.

Mentzer, N. (2011b). High school engineering and technology education integration through design challenges. Journal of STEM Teacher Education, 48(2), 103-136.

Mentzer, N. (2014). Team Based Engineering Design Thinking. Journal of Technology Education, 25(2), 52-72.

Mentzer, N., Becker, K. et Sutton, M. (2015). Engineering design thinking: High school students' performance and knowledge. Journal of Engineering Education, (4), 417-432.

Moore, P. L., Atman, C. J., Bursic, K. M., Shuman, L. J. et Gottfried, B. S. (1995, 25-29 juin). Do freshmen design texts adequately define the engineering design process. American Society for Engineering Education Annual Conference, Ahaheim, California.

Moore, T. J., Stohlmann, M. S., Wang, H. H., Tank, K. M., Glancy, A. W. et Roehrig, G. H. (2014). Implementation and integration of engineering in K-12 STEM education. Dans S. Purzer, J. Strobel et M. E. Cardella (dir.), Engineering in pre-college settings: Synthesizing research, policy, and practices (p. 35-60). Purdue University Press.

Oakes, W. C., Leone, L. L. et Gunn, C. J. (2002). Engineering your future : a comprehensive approach. Great Lakes Press.

Orange, C. (2018, 20 avril). Problématisation et pratiques de savoirs à l'école : Un point de vue développemental. Dans l'UR EADS (Hep. Fribourg) et le LirEDD (HEP Vaud) (resp.), De la problématisation disciplinaire en sciences de la nature à la problématisation interdisciplinaire dans le champ de l'éducation en vue d'un développement durable: quels enjeux, quelles conditions et quelles modalités opératoires possibles? [Séminaire de recherche]. Haute école pédagogique Fribourg, Suisse.

Parkinson, E. (2007). Practical modelling and hypothesis testing in primary design and technology education. International Journal of Technology and Design Education, 17(3), 233-251.

Pearson, G. et Young, A. T. (2002). Technically speaking: Why all Americans need to know more about technology. National Academy of Engineering.

Petroski, H. (1996). Invention by design: How engineers get from thought to thing. Harvard University Press.

Prince, M. J. et Felder, R. M. (2006). Inductive teaching and learning methods : Definitions, comparisons, and research bases. Journal of engineering education, 95(2), 123-138.

Rabardel, P. et Weill-Fassina, A. (1987). Le dessin technique : apprentissage, utilisation, évolution. Hermès.

Rauth, I., Köppen, E., Jobst, B. et Meinel, C. (2010). Design thinking: An educational model towards creative confidence. DS 66-2: Proceedings of the $1^{\text {st }}$ international conference on design creativity (ICDC 2010).

Rohaan, E. J., Taconis, R. et Jochems, W. M. (2010). Reviewing the relations between teachers' knowledge and pupils' attitude in the field of primary technology education. International journal of technology and design education, 20(1), 15.26.

Rohaan, E. J., Taconis, R. et Jochems, W. M. (2011). Exploring the underlying components of primary school teachers' pedagogical content knowledge for technology education. Eurasia Journal of Mathematics, Science and Technology Education, 7(4), $293-304$.

Rohaan, E. J., Taconis, R. et Jochems, W. M. (2012). Analysing teacher knowledge for technology education in primary schools. International Journal of Technology and Design Education, 22(3), 271-280.

Roqueplo, P. (1983). Penser la technique : pour une démocratie concrète. Seuil.

Roth, W.-M. (2001). Learning science through technological design. Journal of Research in Science Teaching : The Official Journal of the National Association for Research in Science Teaching, 38(7), 768-790.

Roy, P. et Bousadra, F. (2017). Peut-on former à une pensée technologique dans l'enseignement général dans le contexte d'un programme intégré des sciences et technologies? Bulletin du Centre de recherche sur l'enseignement et l'apprentissage des sciences (CREAS), (3), 15-19.

Rynearson, A. M. (2016). From mechanic to designer: Evolving perceptions of elementary students over three years of engineering instruction [Thèse de doctorat]. Purdue University.

Sanders, M. E. (2008). Stem, stem education, stemmania. Technology Teacher, 68(4), 20-26.

Schütze, M., Sachse, P. et Römer, A. (2003). Support value of sketching in the design process. Research in Engineering Design, $14(2), 89-97$.

Séris, J.-P. (1994). La technique. Presses universitaires de France. 
Sheppard, S. D., Macatangay, K., Colby, A. et Sullivan, W. M. (2009). Educating Engineers : Designing for the Future of the Field. Book Highlights. Jossey-Bass.

Simon, H. A. (1995) Problem forming, problem finding and problem solving in design. Dans A. Collen et W. Gasparski (dir.), Design et Systems (p. 245-257). Transaction Publishers.

Simondon, G. (1958). Du mode d'existence des objets techniques. Aubier.

Smith, P. C. (2006). Essential aspects and related academic concepts of an engineering design curriculum in secondary technology education [Thèse de doctorat]. University of Georgia.

Trevelyan, J. (2010). Reconstructing engineering from practice. Engineering Studies, 2(3), 175-195.

Van Driel, J. H., Beijaard, D. et Verloop, N. (2001). Professional development and reform in science education : The role of teachers' practical knowledge. Journal of Research in Science Teaching : The Official Journal of the National Association for Research in Science Teaching, 38(2), 137-158.

Vinck, D. (2003). Everyday engineering : An ethnography of design and innovation. MIT Press.

Walker, E. N. (2007). Rethinking professional development for elementary mathematics teachers. Teacher Education Quarterly, 34(3), 113-134.

Welch, M. et Lim, H. S. (2000). The Strategic Thinking of Novice Designers : Discontinuity between Theory and Practice. Journal of Technology Studies, 26(2), 34-44.

Wenger, E. (1998). Communities of practice : learning, meaning, and identity. Cambridge University Press.

Wicklein, R. C. (2006). Five good reasons for engineering as the focus for technology education. The Technology Teacher, 65(7), 25-29.

Wickman, P.-O. (2004). The practical epistemologies of the classroom : A study of laboratory work. Science education, 88(3), 325-344.

Wilson, A. A., Householder, D. L. et Smith, E. (2013). High school students' cognitive activity while solving authentic problems through engineering design processes. Conference Proceedings of the American Society for Engineering Education, 2-37.

Woodman, R. W., Sawyer, J. E., et Griffin, R. W. (1993). Toward a theory of organizational creativity. Academy of management review, 18(2), 293-321.

Mots-clés : Technologie ; processus de conception technique ; pensée design ; pratiques sociotechniques de référence ; pratiques épistémiques d'ingénierie ; communauté discursive disciplinaire scolaire ; école obligatoire

\section{Epistemische Zugänge zum ingenieurwissenschaftlichen Designprozess zur Schaffung einer interdisziplinären diskursiven Gemeinschaft in der obligatorischen Schule}

Zusammenfassung

Ziel dieses Artikels ist eine erkenntnistheoretische und didaktisch konzeptionelle Reflexion über die Ingenieursausbildung vorzunehmen, in deren Mittelpunkt der Prozess des Designs steht. Um eine interdisziplinäre schulische Gemeinschaft in diesem Bereich aufzubauen, laden wir Lehrerinnen und Lehrer ein, den Schülerinnen und Schülern sechs erkenntnisorientierte Arbeitsweisen zu vermitteln: Definieren eines technischen Problems, Generieren und Auswählen von Lösungen unter Verwendung eines systematischen und iterativen Ansatzes, Analysieren und Modellieren, Experimentieren und Auswerten, Kommunizieren und Treffen von Entscheidungen. Diese interdisziplinären diskursiven Praktiken, die das Designdenken der Studierenden charakterisieren, stehen im Einklang mit soziotechnischen Referenzpraktiken und spielen eine zentrale Rolle bei der Entwicklung einer authentischen technischen Kultur.

Schlagworte: Technik; technischer Entwurfsprozess; soziotechnische Referenzpraxis; epistemische Ingenieurspraxis; technisches Denken; disziplinäre Diskursgemeinschaft; Schulpflicht 


\title{
Pratiche epistemiche di ingegneria sul processo di progettazione ingegneristica per istituire una comunità discorsiva interdisciplinare nella scuola dell'obbligo
}

\author{
Riassunto
}

Questo articolo consiste in una riflessione concettuale d'ordine epistemologico e didattico sull'insegnamento della tecnologia incentrato sul processo di progettazione ingegneristica. In vista della costituzione di una comunità discorsiva interdisciplinare scolastica in questo settore, invitiamo gli e le insegnanti a far acquisire agli studenti e alle studentesse sei pratiche epistemiche di ingegneria: definire un problema tecnico, generare e selezionare soluzioni con un approccio sistematico e iterativo, analizzare e modellizzare, sperimentare e valutare, comunicare e prendere decisioni. Queste pratiche discorsive interdisciplinari che caratterizzano il pensiero progettuale degli studenti e delle studentesse sono coerenti con le pratiche sociotecniche di riferimento e svolgono un ruolo centrale nello sviluppo di un'autentica cultura tecnica.

Parole chiave: Tecnologia; processo di progettazione ingegneristica; pratiche sociotecniche di riferimento; pratiche epistemiche di ingegneria; pensiero tecnico; comunità di discorso disciplinare; scuola dell'obbligo

\section{Epistemic engineering practices regarding the engineering design process for an interdisciplinary discursive community in compulsory schooling}

\section{Summary}

This article consists of an epistemological and didactic conceptual reflection on technology education centred on the engineering design process. In order to build a school interdisciplinary discursive community in this field, we invite teachers to teach students six epistemic engineering practices: defining a technical problem, generating and selecting solutions using a systematic and iterative approach, analysing and modelling, experimenting and evaluating, communicating and making decisions. These interdisciplinary discursive practices characterising students' design thinking are coherent with the sociotechnical practices of reference and play a central role in the development of an authentic technical culture.

Keywords: Technology; engineering design process; sociotechnical reference practices; epistemic engineering practices; technical thinking; school discourse community; compulsory schooling

Patrick Roy, PhD. ; doyen de la recherche et prestations à des tiers, responsable de l'Unité de recherche Enseignement et apprentissage des disciplines scientifiques (UR EADS) et professeur HEP en didactiques des sciences. Intérêts de recherche : problématisation et modélisation dans les apprentissages scientifiques, "Éducations à ", interdisciplinarité, analyse et développement des pratiques d'enseignement dans le cadre d'ingénieries didactiques coopératives.

Haute École pédagogique Fribourg, Pädagogische Hochschule Freiburg, Rue de Morat 36, CH-1700 Fribourg, E-Mail: patrick.roy@edufr.ch 
ANNEXE

Tableau 1

Définition des pratiques épistémiques (Cunningham et Kelly, 2017a, p. 2-3)

1. Epistemic practices are interactional ; they are constructed among people through concerted activity. Through discourse processes, members of a group frame opportunities to define what counts as knowledge and knowledge claims.

2. Epistemic practices are contextual. Instances of adjudication of knowledge claims through interactional processes are situated in social practices and cultural norms. For example, disciplines have particular norms for presenting, representing, and assessing knowledge claims (Kelly et al., 2000).

3. Epistemic practice are intertextual. Uses of discourse, signs, and symbols make reference to other discourses, and each is communicated through a history of coherent, interactionally recognized genres of communication.

4. Epistemic practices are consequential. Choices about what counts as reliable, valid, or useful legitimize certain knowledge claims that instantiate power and culture.

\section{Tableau 2}

Comparaison d'un PCT d'ingénieur.e.s avec un PCT pour la classe (Hailey et al., 2005, p. 25)

\begin{tabular}{|c|c|}
\hline $\begin{array}{c}\text { Classical Engineering Design Process } \\
\text { (from introductory engineering text by } \\
\text { Eide et al. (2002)) }\end{array}$ & $\begin{array}{c}\text { Grades 9-12 Design Process } \\
\text { (from Standards for Technological Literacy } \\
\text { (Hailey et al., 2005)) }\end{array}$ \\
\hline Identify the Need & Defining a problem \\
Define the Problem & Brainstorming \\
Search for Solutions & Researching and generating ideas \\
Identify Constraints & Identifying criteria and specifying constraints \\
Execify evaluation criteria & Exploring possibilities \\
Generate alternative solutions & Making a model or prototype \\
Engineering Analysis & Selecting an approposal \\
Optimization & Testing and evaluating the design using specifications \\
Decision & Refining the design \\
Design specifications & Creating or making it \\
Communication & Communicating processes and results \\
\hline
\end{tabular}

\section{Tableau 3}

Quelques caractéristiques d'un cahier des charges fonctionnel

1. Il est construit en amont de la conception et la fabrication d'un objet technique.

2. Il exprime un besoin à satisfaire (nécessaire, réalisable et vérifiable) en termes de fonctions globales et techniques, de contraintes (techniques, réglementaires, budgétaires, etc.) et de spécifications.

3. Il indique un ou des objectif(s) de performance à atteindre et des niveaux de qualité associés aux fonctions à remplir.

4. Il laisse place à la créativité et aux initiatives personnelles du concepteur ou de la conceptrice en ne fournissant pas les solutions techniques liées aux fonctions définies.

5. Sa finalité est d'optimiser le couple besoin-solution.

6. Il comporte des analyses préalables de l'objet technique présentées notamment sous la forme de modélisation graphique.

7. Il est adaptable selon l'évolution du projet.

\section{Tableau 4}

Six types de langages utilisés dans les PCT selon Dym et al. (2005, p. 108)

1. Verbal or textual statements used to articulate design projects, describe objects, describe constraints or limitations, communicate between different members of design and manufacturing teams, and document completed designs ;

2. Graphical representations used to provide pictorial descriptions of designed artifacts such as sketches, renderings, and engineering drawings ;

3. Shape grammars used to provide formal rules of syntax for combining simpler shapes into more complex shapes ;

4. Features used to aggregate and specialize specified geometrical shapes that are often identified with specific functions ;

5. Mathematical or analytical models used to express some aspect of an artifact's function or behavior, where this behaviour is in turn often derived from some physical principle(s); and

6. Numbers used to represent discrete-valued design information (e.g., part dimensions) and parameters in design calculations or within algorithms representing a mathematical model. 